\title{
Supersymmetric gauge theories with higher derivatives and nonlocal terms in the matter sector
}

\author{
F. S. Gama๑, ${ }^{*}$ J. R. Nascimento, ${ }^{\dagger}$ and A. Yu. Petrov $\odot^{*}$ \\ Departamento de Física, Universidade Federal da Paraíba, Caixa Postal 5008, \\ 58051-970 João Pessoa, Paraíba, Brazil
}

(Received 22 April 2020; accepted 14 May 2020; published 26 May 2020)

\begin{abstract}
In this work, we consider local and nonlocal higher-derivative generalizations of the super-ChernSimons theory and four-dimensional supersymmetric QED. In contrast to previous studies, the models studied here also have higher-derivative terms in the matter sector. For these models, we calculate the oneloop superfield effective potential.
\end{abstract}

DOI: $10.1103 /$ PhysRevD.101.105018

\section{INTRODUCTION}

Historically, higher-derivative theories have been introduced in an attempt to prevent singularities in a classical field theory [1], and to avoid ultraviolet divergences of a quantum field theory [2]. In supersymmetric models, higher-derivative theories have been studied within different contexts. For example, the phenomenological implications of an extension of the minimal supersymmetric Standard Model with dimension-five and six operators were investigated in Ref. [3]. In Ref. [4] supersymmetric versions of cubic and quartic Galileon theories were proposed. Nonlocal higher-derivative extensions for the scalar, super Yang-Mills, and supergravity theories were constructed in Ref. [5]. In Ref. [6], a new mechanism to construct ghost-free higher-derivative models was formulated. Recently, the higher covariant derivative regularization, which was first proposed by Slavnov in Ref. [7], was successfully applied in the calculation of the $\beta$ function of supersymmetric gauge theories [8].

The effective potential is an important theoretical tool for studying the ground state of a theory and the phenomena related to it, such as the spontaneous breaking and restoration of symmetries $[9,10]$. In the context of higher-derivative superfield theories, the effective potential has been investigated for different models [11-13]. In particular, in Ref. [14], the one-loop effective potential was explicitly calculated for the simplest higher-derivative

\footnotetext{
*fisicofabricio@yahoo.com.br

jroberto@fisica.ufpb.br

petrov@fisica.ufpb.br
}

Published by the American Physical Society under the terms of the Creative Commons Attribution 4.0 International license. Further distribution of this work must maintain attribution to the author(s) and the published article's title, journal citation, and DOI. Funded by SCOAP. extension of an Abelian gauge superfield theory. In Refs. [15,16], the effective potential was studied in higher-derivative gauge superfield theories defined on the $\mathcal{N}=1$ and $\mathcal{N}=2$ three-dimensional superspaces. More recently, a nonlocal higher-derivative extension of the supersymmetric gauge theory was proposed and the one-loop Kählerian effective potential was explicitly calculated for this theory [17].

One important limitation of the higher-derivative gauge superfield theories studied in Refs. [14-17] is that they do not include higher derivatives in the matter sector. In particular, the four-dimensional theories studied in Refs. $[14,17]$ also do not contain chiral self-interaction terms. Since these terms give nontrivial contributions to the one-loop superfield effective potential, there is no reason (other than convenience for calculating the oneloop effective potential) to ignore higher-derivative and chiral self-interaction terms in the matter sector of a higher-derivative supersymmetric gauge theory. Thus, the aim of this paper is to formulate higher-derivative or nonlocal gauge-covariant terms in the matter sector and to calculate the superfield effective potential at the one-loop level for local and nonlocal higher-derivative generalizations of the super-Chern-Simons theory and supersymmetric QED (SQED) by taking into account these new terms in the matter sector. In this regard, our work is a further development of the studies presented in Refs. [14-17].

This paper is organized as follows. In Sec. II, we formulate a generic higher-derivative super-Chern-Simons theory coupled to matter and calculate the one-loop contribution to the superfield effective potential. In Sec. III, we formulate a generic higher-derivative four-dimensional SQED and explicitly calculate the one-loop Kählerian effective potential in it. In Sec. IV, we give a short summary of the results obtained and suggest a possible continuation of this study. 


\section{HIGHER-DERIVATIVE SUPER-CHERN- SIMONS THEORY}

Our starting point is the following $\mathcal{N}=1, d=3$ higherderivative action for the complex scalar multiplet:

$$
\begin{aligned}
S_{\mathrm{HM}}= & \frac{1}{2} \int d^{5} z\left[\bar{\Phi}\left(f(\square) D^{2}+m g(\square)\right) \Phi+\text { H.c. }\right] \\
& +\int d^{5} z V(\bar{\Phi} \Phi),
\end{aligned}
$$

which is invariant under the global transformations $\delta \Phi=$ $i K \Phi$ and $\delta \bar{\Phi}=-i K \bar{\Phi}$. This model was originally proposed in Ref. [12] and studied only in the context of local theories. The dimensionless operators $f(\square)$ and $g(\square)$ are assumed to be analytical functions of the d'Alembertian operator. Additionally, in order to reproduce the standard action for the complex scalar multiplet, we also suppose that $f(\square)$ and $g(\square)$ coincide with the unit operator in some suitable limit.

We are interested in the coupling of the theory (1) to the Abelian gauge superfield $A_{\alpha}$. In order to do this, we will use the identity $\square=\left(D^{2}\right)^{2}$ and apply the minimal coupling prescription [18], exchanging the simple covariant derivative with the gauge covariant one through the rule

$$
D_{\alpha} \Phi \rightarrow \nabla_{\alpha} \Phi \equiv D_{\alpha} \Phi-i A_{\alpha} \Phi .
$$

Thus, Eq. (1) can be rewritten as

$$
\begin{aligned}
S_{\mathrm{HM}}= & \frac{1}{2} \int d^{5} z\left[\bar{\Phi}\left(f\left(\nabla^{4}\right) \nabla^{2}+m g\left(\nabla^{4}\right)\right) \Phi+\text { H.c. }\right] \\
& +\int d^{5} z V(\bar{\Phi} \Phi) .
\end{aligned}
$$

Evidently, this gauged model is invariant under the local transformations

$$
[\Phi]^{\prime}=e^{i K} \Phi, \quad\left[A_{\alpha}\right]^{\prime}=A_{\alpha}+D_{\alpha} K
$$

Since $A_{\alpha}$ is a nondynamical superfield in Eq. (3), to introduce a consistent dynamics for it we will add to Eq. (3) the following higher-derivative generalization of the supersymmetric Chern-Simons theory:

$$
S_{\mathrm{HCS}}=\frac{1}{2 e^{2}} \int d^{5} z A^{\alpha} h(\square) D^{\beta} D_{\alpha} A_{\beta},
$$

which is also invariant under the transformations (4).

Finally, the higher-derivative version of the super-ChernSimons theory coupled to matter superfields that we will study in this work has the following action:

$$
S=S_{\mathrm{HCS}}+S_{\mathrm{HM}}+S_{\mathrm{GF}},
$$

where, to perform quantum calculations, we conveniently added the gauge-fixing functional

$$
S_{\mathrm{GF}}=\frac{1}{2 e^{2} \alpha} \int d^{5} z A^{\alpha} h(\square) D_{\alpha} D^{\beta} A_{\beta} .
$$

In order to carry out the calculation of the one-loop superfield effective potential in three dimensions [19], we employ the background field method [20]. Making the background-quantum splitting $\Phi \rightarrow \Phi+\phi$ in Eq. (6), assuming that the background superfield satisfies the condition $D_{\alpha} \Phi=0$, and expanding the action to up to the second order in the quantum superfields, after some tedious but straightforward manipulations we obtain

$$
S_{2}=\int d^{5} z\left(\frac{1}{2} A^{\alpha} \hat{H}_{\alpha}{ }^{\beta} A_{\beta}+A^{\alpha} \mathcal{F}_{\alpha}\right)+\frac{1}{2} \int d^{5} z \boldsymbol{\phi}^{T} \hat{O} \boldsymbol{\phi},
$$

where

$$
\begin{aligned}
\hat{H}_{\alpha}^{\beta} & \equiv \frac{h(\square)}{e^{2}}\left(D^{\beta} D_{\alpha}+\frac{1}{\alpha} D_{\alpha} D^{\beta}\right)+\frac{|\Phi|^{2}}{2}\left(-D^{\beta} D_{\alpha}+f(\square) D_{\alpha} D^{\beta}\right) \frac{D^{2}}{\square}-\frac{m|\Phi|^{2}}{2} \frac{[g(\square)-1]}{\square} D_{\alpha} D^{\beta}, \\
\mathcal{F}_{\alpha} & \equiv \frac{i}{2}\left\{\Phi f(\square) D_{\alpha} \bar{\phi}-\bar{\Phi} f(\square) D_{\alpha} \phi+m \Phi \frac{[g(\square)-1]}{\square} D_{\alpha} D^{2} \bar{\phi}-m \bar{\Phi} \frac{[g(\square)-1]}{\square} D_{\alpha} D^{2} \phi\right\},
\end{aligned}
$$

and

$$
\boldsymbol{\phi} \equiv\left(\begin{array}{c}
\phi \\
\bar{\phi}
\end{array}\right), \hat{O} \equiv\left(\begin{array}{cc}
V_{\Phi \Phi} & f(\square) D^{2}+m g(\square)+V_{\Phi \bar{\Phi}} \\
f(\square) D^{2}+m g(\square)+V_{\Phi \bar{\Phi}} & V_{\bar{\Phi} \bar{\Phi}}
\end{array}\right) .
$$

For present purposes, it is useful to diagonalize Eq. (8). To do this, let us consider the following nonlocal change of variables [21]:

$$
A_{\alpha}(z) \rightarrow A_{\alpha}(z)-\int d^{5} z^{\prime} G_{\alpha}^{\beta}\left(z, z^{\prime}\right) \mathcal{F}_{\beta}\left(z^{\prime}\right),
$$


where $G_{\alpha}{ }^{\beta}\left(z, z^{\prime}\right)$ is the Green's function of the operator $\hat{H}_{\alpha}{ }^{\beta}$ defined from the equation

$$
G_{\alpha}^{\beta}\left(z, z^{\prime}\right)=\left(A D^{\beta} D_{\alpha}+B D_{\alpha} D^{\beta}\right) \delta^{5}\left(z-z^{\prime}\right),
$$

where the coefficients $A$ and $B$ are written in the Appendix.

Under the nonlocal transformation (11), the functional (8) assumes the diagonalized form

$$
S_{2}=\frac{1}{2} \int d^{5} z A^{\alpha} \hat{H}_{\alpha}{ }^{\beta} A_{\beta}+\frac{1}{2} \int d^{5} z \boldsymbol{\phi}^{T} \hat{O} \boldsymbol{\phi}-\int d^{5} z d^{5} z^{\prime} \mathcal{F}^{\alpha}(z) G_{\alpha}{ }^{\beta}\left(z, z^{\prime}\right) \mathcal{F}_{\beta}\left(z^{\prime}\right) .
$$

Since Eq. (11) is merely a shift by a constant, it leaves the integration measure in the path integral invariant. By integrating out the quantum superfields $A_{\alpha}$ and $\phi$, we get two contributions to the Euclidean one-loop effective action:

$$
\Gamma^{(1)}=\Gamma_{A}^{(1)}+\Gamma_{\phi}^{(1)} .
$$

The first contribution $\Gamma_{A}^{(1)}$ is given by the trace

$$
\begin{aligned}
\Gamma_{A}^{(1)}= & \frac{1}{2} \operatorname{Tr} \ln \hat{H}_{\alpha}^{\beta}=\frac{1}{2} \operatorname{Tr} \ln \left\{\frac{h(\square)}{e^{2}}\left(D^{\gamma} D_{\alpha}+\frac{1}{\alpha} D_{\alpha} D^{\gamma}\right)\right\}+\frac{1}{2} \operatorname{Tr} \ln \left\{\delta_{\gamma}{ }^{\beta}-\frac{M}{2 \square h(\square)} D^{\beta} D_{\gamma}\right. \\
& \left.-\frac{\alpha M f(\square)}{2 \square h(\square)} D_{\gamma} D^{\beta}+\frac{\alpha m M[g(\square)-1]}{2 \square^{2} h(\square)} D_{\gamma} D^{\beta} D^{2}\right\},
\end{aligned}
$$

where we factored out the inverse of the propagator of $A_{\alpha}$ and defined $M \equiv \frac{1}{2} e^{2}|\Phi|^{2}$.

Since the first trace does not depend on the background superfield, we can drop it. The second trace can be simplified if we assume the Landau gauge $\alpha=0$. Therefore, it follows from Eq. (15) that

$$
\left.\Gamma_{A}^{(1)}\right|_{\alpha=0}=\frac{1}{2} \operatorname{Tr} \ln \left\{\delta_{\gamma}^{\beta}-\frac{M}{2 \square h(\square)} D^{\beta} D_{\gamma}\right\}=\frac{1}{2} \int d^{5} z \int \frac{d^{3} k}{(2 \pi)^{3}} \frac{1}{|k|} \arctan \left[\frac{M}{|k| h\left(-k^{2}\right)}\right] .
$$

We now determine the second contribution $\Gamma_{\phi}^{(1)}$. If we impose the Landau gauge, then $G_{\alpha}{ }^{\beta}\left(z, z^{\prime}\right)=A D^{\beta} D_{\alpha} \delta^{5}\left(z-z^{\prime}\right)$ and the last term in Eq. (13) vanishes due to the identity $D^{\alpha} D_{\beta} D_{\alpha}=0$. Therefore, we can write

$$
\left.\Gamma_{\phi}^{(1)}\right|_{\alpha=0}=-\frac{1}{2} \operatorname{Tr} \ln \hat{O}=-\frac{1}{2} \operatorname{Tr} \ln \left(\begin{array}{cc}
0 & f(\square) D^{2} \\
f(\square) D^{2} & 0
\end{array}\right)-\frac{1}{2} \operatorname{Tr} \ln \left[\hat{I}_{2}+\mathcal{M} \frac{D^{2}}{\square f(\square)}\right],
$$

where we factored out the inverse of the $\phi$ propagator and defined

$$
\mathcal{M} \equiv\left(\begin{array}{cc}
m g(\square)+V_{\bar{\Phi} \Phi} & V_{\bar{\Phi} \bar{\Phi}} \\
V_{\Phi \Phi} & m g(\square)+V_{\bar{\Phi} \Phi}
\end{array}\right) .
$$

Again, we can drop the first trace and the second one can be evaluated to give

$$
\left.\Gamma_{\phi}^{(1)}\right|_{\alpha=0}=\frac{1}{2} \int d^{5} z \int \frac{d^{3} k}{(2 \pi)^{3}} \frac{1}{|k|} \operatorname{Tr} \arctan \left[\frac{\mathcal{M}}{|k| f\left(-k^{2}\right)}\right] .
$$

Finally, substituting Eqs. (16) and (19) into Eq. (14), we can infer that the superfield effective potential is given by the expression

$$
K^{(1)}(\Phi, \bar{\Phi})=\frac{1}{2} \int \frac{d^{3} k}{(2 \pi)^{3}} \frac{1}{|k|}\left\{\arctan \left[\frac{M}{|k| h\left(-k^{2}\right)}\right]+\sum_{i=+,-} \arctan \left[\frac{\lambda_{i}\left(-k^{2}\right)}{|k| f\left(-k^{2}\right)}\right]\right\}
$$

where the $\lambda$ 's are the eigenvalues of the matrix $\mathcal{M}$ :

$$
\lambda_{ \pm}\left(-k^{2}\right)=m g\left(-k^{2}\right)+V_{\bar{\Phi} \Phi} \pm\left(V_{\bar{\Phi} \bar{\Phi}} V_{\Phi \Phi}\right)^{\frac{1}{2}} .
$$


The last step of our calculation is to evaluate the integrals in Eq. (20). However, to evaluate these integrals one must specify $f\left(-k^{2}\right), g\left(-k^{2}\right)$, and $h\left(-k^{2}\right)$. In this work, we will examine two higher-derivative models which lead to an improved ultraviolet behavior of the theory: one local model and one nonlocal model.

A simple local higher-derivative model is defined by

$$
f\left(\nabla^{4}\right)=g\left(\nabla^{4}\right)=1-\frac{\nabla^{4}}{\Lambda_{\mathrm{L}}^{2}}, \quad h(\square)=1-\frac{\square}{\Lambda_{\mathrm{L}}^{2}},
$$

where $\Lambda_{\mathrm{L}}$ is the mass scale at which the higher-derivative contributions begin to be pertinent. It follows from Eq. (22) that

$$
f\left(-k^{2}\right)=g\left(-k^{2}\right)=h\left(-k^{2}\right)=1+\frac{k^{2}}{\Lambda_{\mathrm{L}}^{2}}
$$

All integrals in this article are evaluated approximately by employing the strategy of expansion by regions [22]. Therefore, substituting Eq. (23) into Eq. (20) and assuming that $\Lambda_{\mathrm{L}}$ is large, we find

$$
\begin{aligned}
K_{\mathrm{L}}^{(1)}(\Phi, \bar{\Phi}) \approx-\frac{M^{2}}{16 \pi}(1 & \left.+2 \frac{M^{2}}{\Lambda_{\mathrm{L}}^{2}}+7 \frac{M^{4}}{\Lambda_{\mathrm{L}}^{4}}+30 \frac{M^{6}}{\Lambda_{\mathrm{L}}^{6}}+\cdots\right)-\sum_{i=+,-} \frac{\left(m+\tilde{\lambda}_{i}\right)^{2}}{16 \pi}\left[1+2 \frac{\tilde{\lambda}_{i}\left(m+\tilde{\lambda}_{i}\right)}{\Lambda_{\mathrm{L}}^{2}}\right. \\
& \left.+\frac{\tilde{\lambda}_{i}\left(m+\tilde{\lambda}_{i}\right)^{2}\left(2 m+7 \tilde{\lambda}_{i}\right)}{\Lambda_{\mathrm{L}}^{4}}+2 \frac{\tilde{\lambda}_{i}\left(m+\tilde{\lambda}_{i}\right)^{3}\left(m^{2}+9 m \tilde{\lambda}_{i}+15 \tilde{\lambda}_{i}^{2}\right)}{\Lambda_{\mathrm{L}}^{6}}+\cdots\right],
\end{aligned}
$$

where $\tilde{\lambda}_{ \pm}=V_{\bar{\Phi} \Phi} \pm\left(V_{\bar{\Phi} \bar{\Phi}} V_{\Phi \Phi}\right)^{\frac{1}{2}}$.

On the other hand, a simple nonlocal model is defined by

$$
f\left(\nabla^{4}\right)=g\left(\nabla^{4}\right)=\exp \left(-\frac{\nabla^{4}}{\Lambda_{\mathrm{NL}}^{2}}\right), \quad h(\square)=\exp \left(-\frac{\square}{\Lambda_{\mathrm{NL}}^{2}}\right),
$$

where, similar to the local model, $\Lambda_{\mathrm{NL}}$ describes the characteristic energy at which the nonlocal contributions become important. According to Eq. (25), we have

$$
f\left(-k^{2}\right)=g\left(-k^{2}\right)=h\left(-k^{2}\right)=\exp \left(\frac{k^{2}}{\Lambda_{\mathrm{NL}}^{2}}\right) .
$$

Therefore, substituting Eq. (26) into Eq. (20) and assuming that $\Lambda_{\mathrm{NL}}$ is large, we get

$$
\begin{aligned}
K_{\mathrm{NL}}^{(1)}(\Phi, \bar{\Phi}) \approx & -\frac{M^{2}}{16 \pi}\left(1+2 \frac{M^{2}}{\Lambda_{\mathrm{NL}}^{2}}+6 \frac{M^{4}}{\Lambda_{\mathrm{NL}}^{4}}+\frac{64}{3} \frac{M^{6}}{\Lambda_{\mathrm{NL}}^{6}}+\cdots\right) \\
& -\sum_{i=+,-} \frac{\left(m+\tilde{\lambda}_{i}\right)^{2}}{16 \pi}\left[1+2\left(m+\tilde{\lambda}_{i}\right)\right. \\
& \left.\times \frac{\tilde{\lambda}_{i}}{\Lambda_{\mathrm{NL}}^{2}}+\frac{\tilde{\lambda}_{i}\left(m+\tilde{\lambda}_{i}\right)^{2}\left(m+6 \tilde{\lambda}_{i}\right)}{\Lambda_{\mathrm{NL}}^{4}}+\frac{1}{3} \frac{\tilde{\lambda}_{i}\left(m+\tilde{\lambda}_{i}\right)^{3}\left(m^{2}+23 m \tilde{\lambda}_{i}+64 \tilde{\lambda}_{i}^{2}\right)}{\Lambda_{\mathrm{NL}}^{6}}+\cdots\right] .
\end{aligned}
$$

Since $\Lambda_{\mathrm{L}}$ and $\Lambda_{\mathrm{NL}}$ are finite physical parameters, the oneloop effective potentials (24) and (27) are UV finite. Notice that this finiteness remains even if we set the parameters to be infinitely large, $\Lambda_{\mathrm{L}} \rightarrow \infty$ and $\Lambda_{\mathrm{NL}} \rightarrow \infty$, while many higher-derivative or nonlocal theories turn out to be divergent in this limit which is equivalent to removing the higher-derivative term. Indeed, such one-loop finiteness is a characteristic feature of the three-dimensional theories. Moreover, we note that the expressions (24) and (27) coincide up to the orders $\Lambda_{\mathrm{L}}^{-2}$ and $\Lambda_{\mathrm{NL}}^{-2}$ in the approximations. This coincidence occurs because the operators (22) and (25) are identical in this particular order.

\section{HIGHER-DERIVATIVE SQED}

In the present section, we are interested in a more realistic theory. Thus, let us consider the four-dimensional matter action

$$
\begin{aligned}
S_{M}= & \int d^{8} z\left(\bar{\Phi}_{+} \Phi_{+}+\bar{\Phi}_{-} \Phi_{-}\right) \\
& +\left[\int d^{6} z\left(m \Phi_{-} \Phi_{+}+W\left(\Phi_{-} \Phi_{+}\right)\right)+\text {H.c. }\right],
\end{aligned}
$$

which is invariant under the rigid $U(1)$ transformations 


$$
\left[\Phi_{+}\right]^{\prime}=e^{i \lambda} \Phi_{+}, \quad\left[\Phi_{-}\right]^{\prime}=e^{-i \lambda} \Phi_{-}, \quad\left[\bar{\Phi}_{+}\right]^{\prime}=e^{-i \lambda} \bar{\Phi}_{+}, \quad\left[\bar{\Phi}_{-}\right]^{\prime}=e^{i \lambda} \bar{\Phi}_{-} .
$$

A natural higher-derivative generalization of this model is

$$
\begin{aligned}
S_{\mathrm{HM}}= & \frac{1}{2} \int d^{8} z\left(\bar{\Phi}_{+} f_{+}(\square) \Phi_{+}+\overline{\left(f_{+}(\square) \Phi_{+}\right)} \Phi_{+}+\bar{\Phi}_{-} f_{-}(\square) \Phi_{-}\right. \\
& \left.+\overline{\left(f_{-}(\square) \Phi_{-}\right)} \Phi_{-}\right)+\left[\int d^{6} z\left(m \Phi_{-} g(\square) \Phi_{+}+W\left(\Phi_{-} \Phi_{+}\right)\right)+\text {H.c. }\right],
\end{aligned}
$$

where again $f_{+}(\square), f_{-}(\square)$, and $g(\square)$ are dimensionless analytical functions and coincide with the identity in some suitable limit. This model is essentially a two-superfield version of the one proposed in Ref. [13], which was studied only in the context of nonlocal theories.

Due to the chirality of the superfields $\Phi_{ \pm}$, we have $\bar{D}^{2} D^{2} \Phi_{ \pm}=\square \Phi_{ \pm}$. Thus, the action (30) can be rewritten in a more convenient form,

$$
\begin{aligned}
S_{\mathrm{HM}}= & \frac{1}{2} \int d^{8} z\left(\bar{\Phi}_{+} f_{+}\left(\bar{D}^{2} D^{2}\right) \Phi_{+}+\overline{\left(f_{+}\left(\bar{D}^{2} D^{2}\right) \Phi_{+}\right)} \Phi_{+}+\bar{\Phi}_{-} f_{-}\left(\bar{D}^{2} D^{2}\right) \Phi_{-}\right. \\
& \left.+\overline{\left(f_{-}\left(\bar{D}^{2} D^{2}\right) \Phi_{-}\right)} \Phi_{-}\right)+\left[\int d^{6} z\left(m \Phi_{-} g\left(\bar{D}^{2} D^{2}\right) \Phi_{+}+W\left(\Phi_{-} \Phi_{+}\right)\right)+\text {H.c. }\right] .
\end{aligned}
$$

In order to extend the transformations (29) to local $U(1)$ transformations, we define

$$
\left[\Phi_{+}\right]^{\prime}=e^{i \Lambda} \Phi_{+}, \quad\left[\Phi_{-}\right]^{\prime}=e^{-i \Lambda} \Phi_{-}, \quad\left[\bar{\Phi}_{+}\right]^{\prime}=e^{-i \bar{\Lambda}} \bar{\Phi}_{+}, \quad\left[\bar{\Phi}_{-}\right]^{\prime}=e^{i \bar{\Lambda}} \bar{\Phi}_{-},
$$

where the local parameter $\Lambda$ is chiral.

To extend Eq. (31) up to a form invariant under Eq. (32), we must use the minimal coupling prescription [23]:

$$
\begin{aligned}
D_{\alpha} \Phi_{ \pm} & \rightarrow \nabla_{\alpha} \Phi_{ \pm} \equiv D_{\alpha} \Phi_{ \pm} \mp i \Gamma_{\alpha} \Phi_{ \pm}, \quad \Gamma_{\alpha} \equiv i D_{\alpha} V, \\
\bar{D}_{\dot{\alpha}} \Phi_{ \pm} & \rightarrow \bar{\nabla}_{\dot{\alpha}} \Phi_{ \pm} \equiv \bar{D}_{\dot{\alpha}} \Phi_{ \pm}=0,
\end{aligned}
$$

where the gauge superfield $V$ and the connection $\Gamma_{\alpha}$ transform as

$$
[V]^{\prime}=V+i(\bar{\Lambda}-\Lambda), \quad\left[\Gamma_{\alpha}\right]^{\prime}=\Gamma_{\alpha}+D_{\alpha} \Lambda .
$$

Therefore, Eq. (31) can be rewritten as

$$
\begin{aligned}
S_{\mathrm{HM}}= & \frac{1}{2} \int d^{8} z\left(\bar{\Phi}_{+} e^{V} f_{+}\left(\bar{\nabla}^{2} \nabla^{2}\right) \Phi_{+}+\overline{\left(f_{+}\left(\bar{\nabla}^{2} \nabla^{2}\right) \Phi_{+}\right)} e^{V} \Phi_{+}+\bar{\Phi}_{-} e^{-V} f_{-}\left(\bar{\nabla}^{2} \nabla^{2}\right) \Phi_{-}\right. \\
& \left.+\overline{\left(f_{-}\left(\bar{\nabla}^{2} \nabla^{2}\right) \Phi_{-}\right)} e^{-V} \Phi_{-}\right)+\left[\int d^{6} z\left(m \Phi_{-} g\left(\bar{\nabla}^{2} \nabla^{2}\right) \Phi_{+}+W\left(\Phi_{-} \Phi_{+}\right)\right)+\text {H.c. }\right] .
\end{aligned}
$$

This model is invariant under the combined transformations (32) and (34). Notice that we introduced the factor $\exp (V)$ to change a $\Lambda$ representation to a $\bar{\Lambda}$ representation of the group [18].

Since $V$ has no kinetic term in Eq. (35), we will add to Eq. (35) the following higher-derivative generalization of the supersymmetric Abelian gauge theory:

$$
S_{\mathrm{HG}}=\frac{1}{16 e^{2}}\left[\int d^{6} z W^{\alpha} h(\square) W_{\alpha}+\int d^{6} \bar{z} \bar{W}^{\dot{\alpha}} h(\square) \bar{W}_{\dot{\alpha}}\right],
$$

where the superfield strengths are expressed in terms of the gauge superfield as

$$
W_{\alpha}=i \bar{D}^{2} D_{\alpha} V, \quad \bar{W}_{\dot{\alpha}}=-i D^{2} \bar{D}_{\dot{\alpha}} V .
$$

Finally, the higher-derivative version of the SQED that we will study in this paper is given by

$$
S=S_{\mathrm{HG}}+S_{\mathrm{HM}}
$$

Here, our goal is to calculate the one-loop correction to the Kählerian effective potential [24]. Thus, as we have done in 
the last section, we will expand Eq. (38) around background superfields:

$$
\Phi_{+} \rightarrow \Phi_{+}+\phi_{+}, \quad \Phi_{-} \rightarrow \Phi_{-}+\phi_{-} .
$$

We will assume that the background superfields are subject to the constraints $D_{\alpha} \Phi_{+}=0$ and $D_{\alpha} \Phi_{-}=0$, and keep only quadratic terms in the quantum fluctuations $\phi_{+}$and $\phi_{-}$. Therefore, after some lengthy algebra, we find

$$
\begin{aligned}
S_{2}= & -\frac{1}{8 e^{2}} \int d^{8} z V \square h(\square) \Pi_{\frac{1}{2}} V+\frac{1}{2} \int d^{8} z\left\{\left|\Phi_{+}\right|^{2} V\left[\Pi_{\frac{1}{2}}+f_{+}(\square) \Pi_{0}\right] V+2 \bar{\Phi}_{+} V f_{+}(\square) \phi_{+}\right. \\
& \left.+2 \Phi_{+} V f_{+}(\square) \bar{\phi}_{+}+2 \bar{\phi}_{+} f_{+}(\square) \phi_{+}\right\}+\frac{1}{2} \int d^{8} z\left\{\left|\Phi_{-}\right|^{2} V\left[\Pi_{\frac{1}{2}}+f_{-}(\square) \Pi_{0}\right] V\right. \\
& \left.-2 \bar{\Phi}_{-} V f_{-}(\square) \phi_{-}-2 \Phi_{-} V f_{-}(\square) \bar{\phi}_{-}+2 \phi_{-} f_{-}(\square) \bar{\phi}_{-}\right\} \\
& -m \int d^{8} z\left\{\Phi_{-} \Phi_{+} V \frac{g(\square)-1}{\square} D^{2} V+\Phi_{-} V \frac{g(\square)-1}{\square} D^{2} \phi_{+}-\Phi_{+} \phi_{-} \frac{g(\square)-1}{\square} D^{2} V+\text { H.c. }\right\} \\
& +\left\{\int d^{6} z\left[m \phi_{-} g(\square) \phi_{+}+\frac{1}{2} \frac{\partial^{2} W}{\partial \Phi_{+}^{2}} \phi_{+}^{2}+\frac{1}{2} \frac{\partial^{2} W}{\partial \Phi_{-}^{2}} \phi_{-}^{2}+\frac{\partial^{2} W}{\partial \Phi_{+} \partial \Phi_{-}} \phi_{+} \phi_{-}\right]+\text {H.c. }\right\},
\end{aligned}
$$

where $\Pi_{\frac{1}{2}}$ and $\Pi_{0}$ are the transverse and longitudinal projection operators, which are defined as

$$
\Pi_{1 / 2}=-\frac{D^{\alpha} \bar{D}^{2} D_{\alpha}}{\square}, \quad \Pi_{0+}=\frac{\bar{D}^{2} D^{2}}{\square}, \quad \Pi_{0-}=\frac{D^{2} \bar{D}^{2}}{\square}, \quad \Pi_{0}=\Pi_{0+}+\Pi_{0-} .
$$

At this step of our calculation, we find it more advantageous to work with unconstrained superfields than chiral superfields. For this reason, we will write the quantum antichiral and chiral superfields as $\phi_{ \pm}=\bar{D}^{2} \psi_{ \pm}$and $\bar{\phi}_{ \pm}=D^{2} \bar{\psi}_{ \pm}$, where $\psi_{ \pm}$and $\bar{\psi}_{ \pm}$are free of differential constraints [25]. However, this replacement introduces a new gauge symmetry, namely, $\delta \psi_{ \pm}=$ $\bar{D}^{\dot{\alpha}} \bar{\omega}_{ \pm \dot{\alpha}}$ and $\delta \bar{\psi}_{ \pm}=D^{\alpha} \omega_{ \pm \alpha}$. Therefore, in order to fix this gauge invariance and the one (34), we will add to Eq. (40) the following gauge-fixing functionals:

$$
\begin{gathered}
S_{\mathrm{GF} 1}=-\frac{1}{8 e^{2} \alpha} \int d^{8} z V \square h(\square) \Pi_{0} V, \\
S_{\mathrm{GF} 2}=\int d^{8} z \bar{\psi}_{+} f_{+}(\square)\left(\bar{D}^{2} D^{2}-D^{\alpha} \bar{D}^{2} D_{\alpha}\right) \psi_{+}, \\
S_{\mathrm{GF} 3}=\int d^{8} z \bar{\psi}_{-} f_{-}(\square)\left(\bar{D}^{2} D^{2}-D^{\alpha} \bar{D}^{2} D_{\alpha}\right) \psi_{-} .
\end{gathered}
$$

Therefore, it follows from Eqs. (40)-(44) that

$$
\tilde{S}_{2} \equiv S_{2}+S_{\mathrm{GF} 1}+S_{\mathrm{GF} 2}+S_{\mathrm{GF} 3}=\int d^{8} z\left(\frac{1}{2} V \hat{H} V+V \mathcal{F}\right)+\frac{1}{2} \int d^{8} z \boldsymbol{\psi}^{T} \hat{O} \boldsymbol{\psi}
$$

where

$$
\begin{aligned}
\hat{H} \equiv & -\frac{1}{4 e^{2}} \square h(\square)\left[\Pi_{\frac{1}{2}}+\frac{1}{\alpha} \Pi_{0}\right]+\left(\left|\Phi_{+}\right|^{2}+\left|\Phi_{-}\right|^{2}\right) \Pi_{\frac{1}{2}}+\left(\left|\Phi_{+}\right|^{2} f_{+}(\square)\right. \\
& \left.+\left|\Phi_{-}\right|^{2} f_{-}(\square)\right) \Pi_{0}-2 m \Phi_{-} \Phi_{+}[g(\square)-1] \frac{D^{2}}{\square}-2 m \bar{\Phi}_{-} \bar{\Phi}_{+}[g(\square)-1] \frac{\bar{D}^{2}}{\square}, \\
\mathcal{F} \equiv & \bar{\Phi}_{+} f_{+}(\square) \bar{D}^{2} \psi_{+}-\bar{\Phi}_{-} f_{-}(\square) \bar{D}^{2} \psi_{-}-m \Phi_{-}[g(\square)-1] \Pi_{0-} \psi_{+} \\
& +m \Phi_{+}[g(\square)-1] \Pi_{0-} \psi_{-}+\text {H.c. },
\end{aligned}
$$

and 


$$
\boldsymbol{\psi} \equiv\left(\begin{array}{l}
\psi_{+} \\
\psi_{-} \\
\bar{\psi}_{+} \\
\bar{\psi}_{-}
\end{array}\right), \quad \hat{O} \equiv\left(\begin{array}{cc}
\hat{W} \bar{D}^{2} & \square \hat{F} \\
\square \hat{F} & \hat{\bar{W}} D^{2}
\end{array}\right)
$$

Last, the matrices $\hat{W}$ and $\hat{F}$ are defined as

$$
\hat{F} \equiv\left(\begin{array}{cc}
f_{+}(\square) & 0 \\
0 & f_{-}(\square)
\end{array}\right), \quad \hat{W} \equiv\left(\begin{array}{cc}
\frac{\partial^{2} W}{\partial \Phi_{+}^{2}} & m g(\square)+\frac{\partial^{2} W}{\partial \Phi_{+} \partial \Phi_{-}} \\
m g(\square)+\frac{\partial^{2} W}{\partial \Phi_{+} \partial \Phi_{-}} & \frac{\partial^{2} W}{\partial \Phi_{-}^{2}}
\end{array}\right)
$$

The mixing terms between the quantum superfields $V$ and $\psi$ can be eliminated by the following nonlocal change of variables in the path integral:

$$
V(z) \rightarrow V(z)-\int d^{8} z^{\prime} G\left(z, z^{\prime}\right) \mathcal{F}\left(z^{\prime}\right)
$$

where $G\left(z, z^{\prime}\right)$ is the Green's function of the operator $\hat{H}=\hat{H}(z)$, namely, $\hat{H} G\left(z, z^{\prime}\right)=\delta^{8}\left(z-z^{\prime}\right)$. This equation has the solution

$$
G\left(z, z^{\prime}\right)=\left(X \Pi_{\frac{1}{2}}+Y \Pi_{0}+Z D^{2}+\bar{Z} \bar{D}^{2}\right) \delta^{8}\left(z-z^{\prime}\right) .
$$

The coefficients $X, Y$, and $Z$ are written in the Appendix.

Therefore, after the change of variables $(50)$, the $\tilde{S}_{2}$ can be put in the diagonalized form

$$
\tilde{S}_{2}=\frac{1}{2} \int d^{8} z V \hat{H} V+\frac{1}{2} \int d^{8} z \boldsymbol{\psi}^{T} \hat{O} \boldsymbol{\psi}-\int d^{8} z d^{8} z^{\prime} \mathcal{F}(z) G\left(z, z^{\prime}\right) \mathcal{F}\left(z^{\prime}\right) .
$$

From $\tilde{S}_{2}$, we can compute the Euclidean one-loop effective action by formally integrating out the superfields $V$ and $\boldsymbol{\psi}$. Therefore, we arrive at

$$
\Gamma^{(1)}=\Gamma_{V}^{(1)}+\Gamma_{\psi}^{(1)}
$$

The first contribution $\Gamma_{V}^{(1)}$ is given by the trace,

$$
\begin{aligned}
\Gamma_{V}^{(1)}= & -\frac{1}{2} \operatorname{Tr} \ln \hat{H}=-\frac{1}{2} \operatorname{Tr} \ln \left\{-\frac{1}{4 e^{2}} \square h(\square)\left[\Pi_{\frac{1}{2}}+\frac{1}{\alpha} \Pi_{0}\right]+\frac{1}{4 e^{2}} M \Pi_{\frac{1}{2}}+\frac{1}{4 e^{2}} \tilde{f}(\square) \Pi_{0}\right. \\
& \left.-\frac{2 m}{4 e^{2}} \tilde{g}(\square) \frac{D^{2}}{\square}-\frac{2 m}{4 e^{2}} \tilde{\bar{g}}(\square) \frac{\bar{D}^{2}}{\square}\right\},
\end{aligned}
$$

where we introduced the definitions

$$
\begin{aligned}
M & \equiv 4 e^{2}\left(\left|\Phi_{+}\right|^{2}+\left|\Phi_{-}\right|^{2}\right), \tilde{f}(\square) \equiv 4 e^{2}\left(\left|\Phi_{+}\right|^{2} f_{+}(\square)+\left|\Phi_{-}\right|^{2} f_{-}(\square)\right), \\
\tilde{g}(\square) & \equiv 4 e^{2} \Phi_{+} \Phi_{-}[g(\square)-1], \quad \tilde{g}(\square) \equiv 4 e^{2} \bar{\Phi}_{+} \bar{\Phi}_{-}[g(\square)-1] .
\end{aligned}
$$

We can factor out the inverse of the $V$ propagator, which is independent of the background superfields, and subsequently drop it. Therefore, it follows from Eq. (54) that

$$
\Gamma_{V}^{(1)}=-\frac{1}{2} \operatorname{Tr} \ln \left\{1-\frac{M}{\square h(\square)} \Pi_{\frac{1}{2}}-\frac{\alpha \tilde{f}(\square)}{\square h(\square)} \Pi_{0}+\frac{2 m \alpha \tilde{g}(\square)}{\square h(\square)} \frac{D^{2}}{\square}+\frac{2 m \alpha \tilde{\bar{g}}(\square)}{\square h(\square)} \frac{\bar{D}^{2}}{\square}\right\} .
$$

This trace assumes its simplest form in the Landau gauge $\alpha=0$. Therefore, in this particular gauge, we find 


$$
\left.\Gamma_{V}^{(1)}\right|_{\alpha=0}=-\frac{1}{2} \operatorname{Tr} \ln \left[1-\frac{M}{\square h(\square)} \Pi_{\frac{1}{2}}\right]=-\int d^{8} z \int \frac{d^{4} p}{(2 \pi)^{4}} \frac{1}{p^{2}} \ln \left[1+\frac{M}{p^{2} h\left(-p^{2}\right)}\right]
$$

Let us move on to the calculation of the second contribution $\Gamma_{\psi}^{(1)}$. Notice that $G\left(z, z^{\prime}\right)=X \Pi_{\frac{1}{2}} \delta^{8}\left(z-z^{\prime}\right)$ in the Landau gauge. Since the Green's function is transversal in this gauge, the last term in Eq. (52) vanishes. Therefore, we can write

$$
\left.\Gamma_{\psi}^{(1)}\right|_{\alpha=0}=-\frac{1}{2} \operatorname{Tr} \ln \hat{O}=-\frac{1}{2} \operatorname{Tr} \ln \left(\begin{array}{cc}
\hat{W} \bar{D}^{2} & \square \hat{F} \\
\square \hat{F} & \hat{\bar{W}} D^{2}
\end{array}\right) .
$$

Again, we can factor out the inverse of the $\psi$ propagator and subsequently drop it from Eq. (58). Thus, we can rewrite Eq. (58) as

$$
\left.\Gamma_{\psi}^{(1)}\right|_{\alpha=0}=-\frac{1}{2} \operatorname{Tr} \ln \left[\hat{I}_{4}+\left(\begin{array}{cc}
0 & \frac{\hat{F}^{-1} \hat{W}}{\square} D^{2} \\
\frac{\hat{F}^{-1} \hat{W}}{\square} \bar{D}^{2} & 0
\end{array}\right)\right] .
$$

Only the even powers in the expansion of the logarithm give nonvanishing contributions to the trace. Therefore, we can show that

$$
\begin{aligned}
\left.\Gamma_{\psi}^{(1)}\right|_{\alpha=0} & =-\frac{1}{4} \operatorname{Tr} \ln \left[\hat{I}_{4}-\left(\begin{array}{cc}
\frac{\hat{F}^{-1} \hat{\hat{W}} \hat{F}^{-1} \hat{W}}{\square} \Pi_{0-} & 0 \\
0 & \frac{\hat{F}^{-1} \hat{W} \hat{F}^{-1} \hat{W}}{\square} \Pi_{0+}
\end{array}\right)\right] \\
& =-\frac{1}{4} \operatorname{Tr} \ln \left(\hat{I}_{2}-\frac{\hat{F}^{-1} \hat{\bar{W}} \hat{F}^{-1} \hat{W}}{\square} \Pi_{0-}\right)-\frac{1}{4} \operatorname{Tr} \ln \left(\hat{I}_{2}-\frac{\hat{F}^{-1} \hat{W} \hat{F}^{-1} \hat{\bar{W}}}{\square} \Pi_{0+}\right) .
\end{aligned}
$$

These traces can be evaluated to give

$$
\left.\Gamma_{\psi}^{(1)}\right|_{\alpha=0}=\frac{1}{2} \int d^{8} z \int \frac{d^{4} p}{(2 \pi)^{4}} \frac{1}{p^{2}} \operatorname{Tr} \ln \left(\hat{I}_{2}+\frac{\hat{F}^{-1} \hat{\bar{W}} \hat{F}^{-1} \hat{W}}{p^{2}}\right) .
$$

This integral is rather complicated. In order to obtain clear analytical results, we will assume that $m=0$ and

$$
f_{+}\left(\bar{\nabla}^{2} \nabla^{2}\right)=f_{-}\left(\bar{\nabla}^{2} \nabla^{2}\right) \equiv f\left(\bar{\nabla}^{2} \nabla^{2}\right) .
$$

Therefore, it follows that

$$
\left.\Gamma_{\psi}^{(1)}\right|_{\alpha=0}=\frac{1}{2} \int d^{8} z \int \frac{d^{4} p}{(2 \pi)^{4}} \frac{1}{p^{2}} \operatorname{Tr} \ln \left(\hat{I}_{2}+\frac{\left.\hat{\bar{W}} \hat{W}\right|_{m=0}}{p^{2} f^{2}\left(-p^{2}\right)}\right)
$$

Finally, substituting Eqs. (57) and (63) into Eq. (53), we can infer that the one-loop correction to the Kähler effective potential is given by

$$
K^{(1)}(\Phi, \bar{\Phi})=\int \frac{d^{4} p}{(2 \pi)^{4}} \frac{1}{p^{2}}\left\{-\ln \left[1+\frac{M}{p^{2} h\left(-p^{2}\right)}\right]+\frac{1}{2} \sum_{i=+,-} \ln \left[1+\frac{\lambda_{i}}{p^{2} f^{2}\left(-p^{2}\right)}\right]\right\},
$$

where the $\lambda$ 's are the eigenvalues of the matrix $\left.\hat{\bar{W}} \hat{W}\right|_{m=0}$ and they are given by

$$
\begin{aligned}
\lambda_{ \pm}= & \frac{1}{2}\left\{\left|\frac{\partial^{2} W}{\partial \Phi_{+}^{2}}\right|^{2}+2\left|\frac{\partial^{2} W}{\partial \Phi_{+} \partial \Phi_{-}}\right|^{2}+\left|\frac{\partial^{2} W}{\partial \Phi_{-}^{2}}\right|^{2} \pm\left[\left(\left|\frac{\partial^{2} W}{\partial \Phi_{+}^{2}}\right|^{2}+\left|\frac{\partial^{2} W}{\partial \Phi_{-}^{2}}\right|^{2}\right)^{2}\right.\right. \\
& \left.\left.+4\left|\frac{\partial^{2} W}{\partial \Phi_{+} \partial \Phi_{-}} \frac{\partial^{2} \bar{W}}{\partial \bar{\Phi}_{+}^{2}}+\frac{\partial^{2} \bar{W}}{\partial \bar{\Phi}_{+} \partial \bar{\Phi}_{-}} \frac{\partial^{2} W}{\partial \Phi_{-}^{2}}\right|^{2}\right]^{\frac{1}{2}}\right\} .
\end{aligned}
$$


In the same manner as in the previous section, it is necessary to specify the functions $f\left(-p^{2}\right)$ and $h\left(-p^{2}\right)$ in order to evaluate the integrals in Eq. (64). Thus, again, we will consider one local and one nonlocal higher-derivative model.

The local higher-derivative model is described by

$$
f\left(\bar{\nabla}^{2} \nabla^{2}\right)=1-\frac{\bar{\nabla}^{2} \nabla^{2}}{\Lambda_{\mathrm{L}}^{2}}, \quad h(\square)=1-\frac{\square}{\Lambda_{\mathrm{L}}^{2}} .
$$

Thus, we can infer that

$$
f\left(-p^{2}\right)=h\left(-p^{2}\right)=1+\frac{p^{2}}{\Lambda_{\mathrm{L}}^{2}}
$$

Therefore, replacing these functions in Eq. (64) and assuming that $\Lambda_{\mathrm{L}}$ is large, we obtain

$$
\begin{aligned}
K_{\mathrm{L}}^{(1)}(\Phi, \bar{\Phi}) \approx & \frac{M}{16 \pi^{2}}\left\{-1+\ln \left(\frac{M}{\Lambda_{\mathrm{L}}^{2}}\right)+\frac{M}{2 \Lambda_{\mathrm{L}}^{2}}\left[1+2 \ln \left(\frac{M}{\Lambda_{\mathrm{L}}^{2}}\right)\right]+\frac{M^{2}}{6 \Lambda_{\mathrm{L}}^{4}}\left[10+12 \ln \left(\frac{M}{\Lambda_{\mathrm{L}}^{2}}\right)\right]\right. \\
& \left.+\frac{M^{3}}{12 \Lambda_{\mathrm{L}}^{6}}\left[59+60 \ln \left(\frac{M}{\Lambda_{\mathrm{L}}^{2}}\right)\right]+\cdots\right\}-\sum_{i=+,-} \frac{\lambda_{i}}{32 \pi^{2}}\left[\ln \left(\frac{\lambda_{i}}{\Lambda_{\mathrm{L}}^{2}}\right)+\frac{\lambda_{i}}{6 \Lambda_{\mathrm{L}}^{2}}[13\right. \\
& \left.\left.+12 \ln \left(\frac{\lambda_{i}}{\Lambda_{\mathrm{L}}^{2}}\right)\right]+\frac{\lambda_{i}^{2}}{20 \Lambda_{\mathrm{L}}^{4}}\left[193+140 \ln \left(\frac{\lambda_{i}}{\Lambda_{\mathrm{L}}^{2}}\right)\right]+\frac{\lambda_{i}^{3}}{84 \Lambda_{\mathrm{L}}^{6}}\left[3825+2520 \ln \left(\frac{\lambda_{i}}{\Lambda_{\mathrm{L}}^{2}}\right)\right]+\cdots\right] .
\end{aligned}
$$

On the other hand, the nonlocal higher-derivative model is described by

$$
f\left(\bar{\nabla}^{2} \nabla^{2}\right)=\exp \left(-\frac{\bar{\nabla}^{2} \nabla^{2}}{\Lambda_{\mathrm{NL}}^{2}}\right), \quad h(\square)=\exp \left(-\frac{\square}{\Lambda_{\mathrm{NL}}^{2}}\right) .
$$

Evidently, Eq. (69) implies that

$$
f\left(-p^{2}\right)=h\left(-p^{2}\right)=\exp \left(\frac{p^{2}}{\Lambda_{\mathrm{NL}}^{2}}\right) .
$$

Therefore, replacing these functions in Eq. (64) and assuming that $\Lambda_{\mathrm{NL}}$ is large, we have

$$
\begin{aligned}
K_{\mathrm{NL}}^{(1)}(\Phi, \bar{\Phi}) \approx & \frac{M}{16 \pi^{2}}\left\{\ln \left(\frac{M}{\Lambda_{\mathrm{NL}}^{2} e^{1-\gamma}}\right)+\frac{M}{\Lambda_{\mathrm{NL}}^{2}} \ln \left(\frac{2 M}{\Lambda_{\mathrm{NL}}^{2} e^{1-\gamma}}\right)+\frac{M^{2}}{4 \Lambda_{\mathrm{NL}}^{4}}\left[-1+6 \ln \left(\frac{3 M}{\Lambda_{\mathrm{NL}}^{2} e^{1-\gamma}}\right)\right]\right. \\
& \left.+\frac{4 M^{3}}{9 \Lambda_{\mathrm{NL}}^{6}}\left[-2+6 \ln \left(\frac{4 M}{\Lambda_{\mathrm{NL}}^{2} e^{1-\gamma}}\right)\right]+\cdots\right\}-\sum_{i=+,-} \frac{\lambda_{i}}{32 \pi^{2}}\left[\ln \left(\frac{2 \lambda_{i}}{\Lambda_{\mathrm{NL}}^{2} e^{1-\gamma}}\right)+\frac{2 \lambda_{i}}{\Lambda_{\mathrm{NL}}^{2}}\right. \\
& \left.\times \ln \left(\frac{4 \lambda_{i}}{\Lambda_{\mathrm{NL}}^{2} e^{1-\gamma}}\right)+\frac{\lambda_{i}^{2}}{\Lambda_{\mathrm{NL}}^{4}}\left[-1+6 \ln \left(\frac{6 \lambda_{i}}{\Lambda_{\mathrm{NL}}^{2} e^{1-\gamma}}\right)\right]+\frac{32 \lambda_{i}^{3}}{288 \Lambda_{\mathrm{NL}}^{6}}\left[-2+6 \ln \left(\frac{8 \lambda_{i}}{\Lambda_{\mathrm{NL}}^{2} e^{1-\gamma}}\right)\right]+\cdots\right] .
\end{aligned}
$$

Like the one-loop effective potentials obtained in the previous section, the Kähler effective potentials (68) and (71) are also UV finite. However, when we set the parameters to be infinitely large $\left(\Lambda_{\mathrm{L}} \rightarrow \infty\right.$ and $\left.\Lambda_{\mathrm{NL}} \rightarrow \infty\right)$ such finiteness ceases to exist because of the leading term of the potentials. We know this must be so because in the limits $\Lambda_{\mathrm{L}} \rightarrow \infty$ and $\Lambda_{\mathrm{NL}} \rightarrow \infty$ both potentials (68) and (71) must agree with the one for the standard SQED.

\section{SUMMARY}

We considered the higher-derivative/nonlocal extensions of supergauge theories where, unlike previous papers on such theories [14-17], the higher derivatives or nonlocality are implemented not only in the gauge sector, but also in the matter sector. It is important to note that within our approach, these kinds of theories are treated within the same methodology. Effectively, we introduced a new class of higher-derivative/nonlocal Abelian supergauge theories and a new class of gauge-matter couplings.

We performed the one-loop calculations in these theories with the use of the functional supertrace approach and explicitly demonstrated that this approach can be applied to these theories with the same degree of success as that for other supergauge theories. In the three-dimensional case, 
the one-loop effective potential is finite and it continues to be so even when the characteristic scale $\Lambda_{\mathrm{L}}$ (or $\Lambda_{\mathrm{NL}}$ ) goes to infinity, as it must be, since the one-loop effective action is finite in three-dimensional theories. This is not so in the four-dimensional case since the one-loop effective potential in the usual SQED diverges [25,26], and the higherderivative/nonlocal terms in various field theory models clearly play the role of the regularization.

It is worth pointing out that, in general, the introduction of higher-derivative terms in a four-dimensional gauge theory renders all multiloop diagrams convergent at finite $\Lambda$, but the one-loop diagrams are still divergent [27]. Despite this, we found that the one-loop effective potential (68) is finite, at finite $\Lambda_{\mathrm{L}}$. This finiteness can be explained by the fact that the supergraphs which contribute to the effective potential are those involving only external matter superfield legs with vanishing external momentum and, as explicitly shown in Ref. [28] for a theory similar to Eq. (38), the higher covariant derivative regularization ensures complete regularization of such one-loop supergraphs.
The net result of our paper consists in the formulation of new gauge-matter couplings. Therefore, it is natural to expect that these couplings can be generalized to other theories, especially to those that are interesting from the phenomenological viewpoint, and to various effective theories. The advantage of such theories consists in the fact that they have better renormalization properties than the conventional ones. We plan to study phenomenological impacts of new couplings in our next papers.

\section{ACKNOWLEDGMENTS}

The work of A. Yu. P. has been partially supported by the CNPq project No. 301562/2019-9.

\section{APPENDIX}

Here we list the coefficients of the Green's functions (12) and (51):

$$
\begin{gathered}
A=-\frac{e^{2}}{2} \frac{e^{2}|\Phi|^{2} D^{2}+2 \square h(\square)}{e^{4}|\Phi|^{4} \square-4 \square^{2} h^{2}(\square)}, \\
B=\frac{e^{2} \alpha}{2} \frac{\alpha e^{2}|\Phi|^{2} f(\square) D^{2}-2 \square h(\square)+\alpha m e^{2}|\Phi|^{2}[g(\square)-1]}{\alpha^{2} e^{4}|\Phi|^{4} \square f^{2}(\square)-\left\{2 \square h(\square)-\alpha m e^{2}|\Phi|^{2}[g(\square)-1]\right\}^{2}}, \\
X=\frac{4 e^{2}}{-\square h(\square)+M}, \\
Y=\frac{4 e^{2} \alpha \square[-\square h(\square)+\alpha \tilde{f}(\square)]}{\square[-\square h(\square)+\alpha \tilde{f}(\square)]^{2}-4 \alpha^{2} m^{2} \tilde{g}(\square) \tilde{\bar{g}}(\square)}, \\
Z=\frac{2 m \alpha \tilde{g}(\square)}{\square(-\square h(\square)+\alpha \tilde{f}(\square))} Y .
\end{gathered}
$$

[1] F. Bopp, Ann. Phys. (Berlin) 430, 345 (1940); B. Podolsky, Phys. Rev. 62, 68 (1942); K. S. Stelle, Gen. Relativ. Gravit. 9, 353 (1978).

[2] T. D. Lee and G. C. Wick, Phys. Rev. D 2, 1033 (1970); K. S. Stelle, Phys. Rev. D 16, 953 (1977).

[3] I. Antoniadis, E. Dudas, D. M. Ghilencea, and P. Tziveloglou, Nucl. Phys. B808, 155 (2009); B831, 133 (2010).

[4] J. Khoury, J. L. Lehners, and B. A. Ovrut, Phys. Rev. D 84, 043521 (2011); M. Koehn, J. L. Lehners, and B. A. Ovrut, Phys. Rev. D 88, 023528 (2013); F. Farakos, C. Germani, and A. Kehagias, J. High Energy Phys. 11 (2013) 045.
[5] T. Kimura, A. Mazumdar, T. Noumi, and M. Yamaguchi, J. High Energy Phys. 10 (2016) 022; S. Giaccari and L. Modesto, Phys. Rev. D 96, 066021 (2017).

[6] T. Fujimori, M. Nitta, and Y. Yamada, J. High Energy Phys. 09 (2016) 106; T. Fujimori, M. Nitta, K. Ohashi, Y. Yamada, and R. Yokokura, J. High Energy Phys. 09 (2017) 143; T. Fujimori, M. Nitta, K. Ohashi, and Y. Yamada, J. High Energy Phys. 05 (2018) 102.

[7] A. A. Slavnov, Nucl. Phys. B31, 301 (1971); Teor. Mat. Fiz. 13, 174 (1972) [Theor. Math. Phys. 13, 1064 (1972)]. 
[8] K. V. Stepanyantz, J. High Energy Phys. 10 (2019) 011; M. D. Kuzmichev, N. P. Meshcheriakov, S. V. Novgorodtsev, I. E. Shirokov, and K. V. Stepanyantz, Eur. Phys. J. C 79, 809 (2019); K. V. Stepanyantz, J. High Energy Phys. 01 (2020) 192.

[9] S. Coleman and E. Weinberg, Phys. Rev. D 7, 1888 (1973).

[10] D. A. Kirzhnits and A. D. Linde, Phys. Lett. 42B, 471 (1972); S. Weinberg, Phys. Rev. D 9, 3357 (1974); L. Dolan and R. Jackiw, Phys. Rev. D 9, 3320 (1974); C. W. Bernard, Phys. Rev. D 9, 3312 (1974).

[11] I. L. Buchbinder and A. Yu. Petrov, Mod. Phys. Lett. A 11, 2159 (1996); Classical Quantum Gravity 14, 21 (1997); M. Gomes, J. R. Nascimento, A. Yu. Petrov, and A. J. da Silva, Phys. Lett. B 682, 229 (2009); F. S. Gama, M. Gomes, J. R. Nascimento, A. Yu. Petrov, and A. J. da Silva, Phys. Lett. B 733, 247 (2014); M. Cvetic, T. Mariz, and A. Yu. Petrov, Phys. Rev. D 92, 085041 (2015).

[12] F. S. Gama, J. R. Nascimento, and A. Yu. Petrov, Phys. Rev. D 88, 065029 (2013).

[13] E. R. Bezerra de Mello, F. S. Gama, J. R. Nascimento, and A. Yu. Petrov, Phys. Rev. D 95, 025028 (2017).

[14] F. S. Gama, M. Gomes, J. R. Nascimento, A. Yu. Petrov, and A. J. da Silva, Phys. Rev. D 84, 045001 (2011).

[15] F. S. Gama, J. R. Nascimento, and A. Yu. Petrov, Phys. Rev. D 88, 045021 (2013).

[16] F. S. Gama, M. Gomes, J. R. Nascimento, A. Yu. Petrov, and A. J. da Silva, Phys. Rev. D 89, 085018 (2014).

[17] F. S. Gama, J. R. Nascimento, A. Y. Petrov, and P. J. Porfirio, Phys. Rev. D 96, 105009 (2017).
[18] S. J. Gates, M. T. Grisaru, M. Rocek, and W. Siegel, Front. Phys. 58, 1 (1983), https://arxiv.org/abs/hep-th/0108200.

[19] A. F. Ferrari, M. Gomes, A. C. Lehum, J. R. Nascimento, A. Y. Petrov, E. O. Silva, and A. J. da Silva, Phys. Lett. B 678, 500 (2009).

[20] B. S. DeWitt, Dynamical Theory of Groups and Fields (Gordon and Breach, New York, 1965).

[21] A. A. Ostrovsky and G. A. Vilkovisky, J. Math. Phys. (N.Y.) 29, 702 (1988); S. M. Kuzenko and S. J. Tyler, J. High Energy Phys. 05 (2007) 081; I. L. Buchbinder and B. S. Merzlikin, Nucl. Phys. B900, 80 (2015).

[22] M. Beneke and V. A. Smirnov, Nucl. Phys. B522, 321 (1998); V. A. Smirnov, Applied Asymptotic Expansions in Momenta and Masses (Springer, New York, 2002).

[23] I. L. Buchbinder and S. M. Kuzenko, Ideas and Methods of Supersymmetry and Supergravity (IOP Publishing, Bristol and Philadelphia, 1995).

[24] I. L. Buchbinder, S. M. Kuzenko, and J. V. Yarevskaya, Nucl. Phys. B411, 665 (1994); Phys. At. Nucl. 56, 680 (1993); https://inspirehep.net/literature/363151.

[25] M. T. Grisaru, M. Rocek, and R. von Unge, Phys. Lett. B 383, 415 (1996).

[26] B. de Wit, M. T. Grisaru, and M. Rocek, Phys. Lett. B 374, 297 (1996); A. Pickering and P. West, Phys. Lett. B 383, 54 (1996).

[27] A. A. Slavnov, Teor. Mat. Fiz. 33, 210 (1977) [Theor. Math. Phys. 33, 977 (1977)].

[28] S. S. Aleshin, A. E. Kazantsev, M. B. Skoptsov, and K. V. Stepanyantz, J. High Energy Phys. 05 (2016) 014. 